\title{
Cardiomyopathies in Tropical Countries: Causes and Nosological Perspective ${ }^{*}$
}

\author{
Jean-Etienne Touze ${ }^{1}$, Laurent Fourcade ${ }^{2}$ \\ ${ }^{1}$ Centre Cardiovasculaire Valmante, ORPEA-Clinea Group, Marseilles, France \\ ${ }^{2}$ Service de cardiologie, Hôpital d’Instruction des Armées Laveran, Marseille, France. \\ Email: jetouze@aol.com; fourcadelaurent@orange.fr
}

Received September 2, 2013; revised October 2, 2013; accepted October 10, 2013

Copyright (C) 2013 Jean-Etienne Touze, Laurent Fourcade. This is an open access article distributed under the Creative Commons Attribution License, which permits unrestricted use, distribution, and reproduction in any medium, provided the original work is properly cited.

\begin{abstract}
Background: Cardiomyopathy is the main cause of heart failure in developing countries, mainly in Africa. In those areas the concept of "tropical cardiomyopathy" is still used to design all unexplained cardiomyopathy. The primary aim of this review is first to review the main etiologies of cardiomyopathies observed in tropical countries and second to gain a better understanding of the nosological place of the so-called "tropical cardiomyopathies" in the current framework of cardiomyopathies. Methods and Results: We reviewed relevant references over the last forty years (June, 1976 to May 2012). Given literature data, endomyocardial fibrosis (EMF) is mainly diagnosed in sub-Saharan countries, as well as Brazil and India. Peripartum cardiomyopathy (PPCM) is observed with a higher prevalence than in temperate countries. Sickle cell anemia does not induce specific cardiomyopathy in all echocardiographic studies. Malnutrition and chronic anemia can induce reversible cardiac dysfunction. Myocardial involvement in parasitic infections is restricted to Chagas disease and probably to human African trypanosomiasis. Helminthiasis is not involved in the pathogenesis of cardiomyopathy except for the deleterious effect of high eosinophilia induced by some endemic diseases (filariasis, schistosomiasis). Primary cardiomyopathies (dilated, hypertrophic, and restrictive cardiomyopathy) have no specificity. Arrhythmogenic right ventricular dysplasia and left ventricular noncompaction are also reported and do not differ from elsewhere. Conclusions: The concept of tropical cardiomyopathy is no longer relevant as most of the cardiomyopathies observed in tropical countries have no specificity, with few exceptions (PPCM, EMF, Chagas disease). In this context, the European Society of Cardiology classification offers a simpler clinical approach and allows the inclusion of the rare tropical specificities.
\end{abstract}

Keywords: Tropical Countries; Cardiomyopathy; Endomyocardial Fibrosis; Peripartum Cardiomyopathy; Chagas Disease

\section{Introduction}

Tropical developing countries are undergoing an epidemiological transition with an alarming rise in coronary disease, hypertension, and diabetes. Meanwhile, despite some hope to control infectious and parasitic diseases, these remain as significant as in the past [1]. In this context, cardiomyopathy, which is the leading cause of cardiac dysfunction in these parts of the world, is a topic of substantial importance. Cardiomyopathies present many challenges to the practitioner. The first one is the lack of adequate diagnostic tests. Invasive techniques, particularly those involving coronary angiography and histology,

"Funding: This work was supported by the ORPEA-CLINEA Group. are not available in most of these countries. The second challenge has to do with the role of infectious and parasitic diseases in the genesis of cardiomyopathy in the tropics. Although the determining role of HIV and opportunistic infections in cardiomyopathy is well established, the same cannot be said of many parasitic diseases for which the pathogenetic mechanism has been extensively reviewed. Furthermore, insufficient resources and limited access to medical care preclude appropriate management, and the chances of transplantation are essentially nil. Moreover, a large number of terms were used to refer to heart failure of unknown etiology in the tropics. "Cardiomyopathy" was initially used to designate all idiopathic heart disease. "Primary parietal en- 
domyocarditis" was favored over endomyocardial fibrosis (EMF) because the fibrous lesions affected the endocardium and the benefits of surgical decortication were undeniable, which would not have been the case if the myocardium had been anatomically injured [2]. The onset of cardiomyopathy has also been attributed to numerous parasitic diseases based on fragmented histological data. This is why the framework of the so-called "tropical" cardiomyopathies prevailed for a long time [3], adding confusion to the cardiomyopathy concept. For all these reasons, and in view of recent advances, the objective of this paper is first to summarize the etiologies of the main cardiomyopathies observed in tropical countries and second to examine whether the concept of "tropical cardiomyopathy" is still relevant in the current framework of cardiomyopathies.

\section{Classification of Cardiomyopathies}

Cardiomyopathies constitute an entity which includes myocardial disorders associated with structural or functional abnormalities of the heart muscle and not associated with hypertension or coronary artery, valvular, or congenital heart disease. For several decades, the nosology of cardiomyopathies remained controversial. Consensus was first achieved in 1995 with the WHO classification [4]. For the first time, nosological clarification began to emerge as two main cardiomyopathy groups were differentiated. Primary cardiomyopathies include dilated, hypertrophic, and restrictive cardiomyopathy (DCM, HCM, and RCM, respectively), as well as arrhythmogenic right ventricular dysplasia (ARVD). Secondary cardiomyopathies include a large number of disorders. There is no question that this classification was helpful, but after a few years it became obsolete because new diseases were identified that could induce fatal ventricular arrhythmias (e.g., Brugada syndrome, long-QT syndrome, short-QT syndrome...). This is why, in 2006, the American Heart Association (AHA) proposed a new classification, which took into account the previous decade's advances by including channelopathies [5].

The new definition of cardiomyopathies, which arose from the AHA experts consensus, is the following: "Cardiomyopathies are a heterogeneous group of diseases of the myocardium associated with mechanical and/or electrical dysfunction that usually (but not invariably) exhibit inappropriate ventricular hypertrophy or dilatation and are due to a variety of causes that frequently are genetic." The AHA distinguishes two major groups of cardiomyopathies. The first group, primary cardiomyopathies, includes the following:

- Genetic cardiomyopathies, represented mostly by HCM, ARVD, left ventricular noncompaction (LVNC), mitochondrial cardiomyopathies, and channelopathies (Brugada syndrome, long-QT and short-QT syndrome, and Coumel syndrome characterized by catecholaminergic polymorphic ventricular tachycardia).

- Mixed cardiomyopathies (genetic or nongenetic) including DCM and RCM (such as EMF).

- Acquired cardiomyopathies, such as inflammatory myocarditis, due to a variety of causes (e.g., viral, bacterial, parasitic, toxic), peripartum cardiomyopathy (PPCM), and the Takotsubo syndrome, associated with stress.

In the second group, secondary cardiomyopathies, myocardial pathology is part of generalized multiorgan disorders. This exhaustive classification includes a large number of diseases, some of which were historically classified as tropical cardiomyopathies.

\section{3. “Tropical” Cardiomyopathies}

\subsection{Endomyocardial Fibrosis (EMF)}

Endomyocardial fibrosis (EMF), which was described by Davies in Uganda in 1948, is an RCM indistinguishable, from an anatomical point of view, from Löeffler's endocarditis which is seen in temperate regions. Both diseases are characterized by scarring of the endocardium and, to a lesser degree, of the myocardium. Löeffler's endocarditis and EMF differ, however, by the context in which they are diagnosed. The former is usually seen in temperate countries. It is consistently associated with substantial eosinophilia, which occurs either as part of an atopy or as a hypereosinophilic syndrome with expected damage to the heart. In contrast, EMF is seen exclusively in tropical countries [6]. Its prevalence is high in Uganda, Nigeria, the Ivory Coast, India, and Brazil. It accounts for $15 \%$ to $20 \%$ of heart failure deaths in these regions of the world. Contrary to Löeffler's endocarditis, EMF is primarily a disease of children and young adults, and its pathogenesis is not fully understood. The high prevalence of EMF in regions where helminth infections with substantial eosinophilia are also prevalent suggests that polynuclear eosinophils play a key role. Yet low levels of polynuclear eosinophils have been found at the time of EMF diagnosis, perhaps because in those cases EMF was a late stage of eosinophilic heart disease that had been caused initially, but a long time before, by a helminth infection $[7,8]$. Other pathogenesis hypotheses include the protein deficiency induced by a cassava-based diet or the role of auto-immune myocarditis, which is supported by high levels of antibodies to myocardial cells in EMF patients [9].

The clinical presentation of EMF depends on the location of the fibrosis. This allows distinction between right ventricular dominant forms with severe tricuspid regurgitation, left ventricular dominant forms characterized by severe mitral regurgitation with pulmonary hypertension, and biventricular forms. The prognosis is poor with a 
mortality rate of $75 \%$ within two years of diagnosis. The symptoms don't respond well to standard heart failure therapy. Corticosteroids and immunosuppressants can help in the early stages of the disease, but surgical endocardectomy with valve repair or replacement is the only alternative that might affect clinical progression. Survival under these conditions is estimated at more than $50 \%$ at 20 years with better results, from a functional point of view, with left heart than right heart dominant disease $[10,11]$.

\subsection{Peripartum Cardiomyopathy (PPCM)}

Peripartum Cardiomyopathy (PPCM) is seen at all latitudes but is more prevalent in sub-Saharan Africa. It occurs between the last month of pregnancy and the five months after delivery, in previously healthy women without any cardiovascular disease (e.g., hypertension, valvular disease) before and during pregnancy. PPCM is rare (1 case/100,000 deliveries in western countries). In contrast, prevalence is high $(>1 / 10,000)$ in tropical Africa. PPCM represents at least $10 \%$ of cardiomyopathies in Australian women and even more in sub-Saharan Africa where there is great geographical disparity. For example, the incidence is 1 case in 1000 deliveries in Nigeria [12]. The etiology and pathogenesis remain unknown, but risk factors have been proposed such as excessive dietary salt intake, selenium deficiency, twin pregnancy, or high parity. Many current studies attribute PPCM to autoimmune processes and excessive prolactin secretion, which would alter the anti-oxidative functions of protein STAT3, which causes cardiac apoptosis [13]. Bromocriptine treatment is consistent with this hypothesis.

Clinical presentation is that of congestive heart failure with severely decreased contractile function. The left ventricular ejection fraction is often less than $40 \%$ with left chamber enlargement, mitral regurgitation due to mitral valve annulus dilatation, and pulmonary edema. Intracardiac thrombus is common. PPCM is associated with complete recovery in $30 \%$ to $50 \%$ of cases or, in more than $30 \%$ of cases, irreversible DCM. Death can occur in $10 \%$ to $30 \%$ of cases during the acute phase. Prognostic factors have been identified, such as the NYHA (New York Heart Association) grades or a high level of C-reactive protein. Recurrence is possible after a subsequent pregnancy, with a much greater risk of progression toward terminal heart failure [14], especially if the heart has not completely recovered after the first episode. As in the case of any DCM, there is a constant risk of hemodynamic complications, arrhythmia, and thrombo-embolism. Management consists of a strict salt-free diet, bed rest, and the standard heart failure medications. This treatment must be administered for at least one year after resolution of the first episode to avoid relapse. The patient should be advised against a subsequent pregnancy.

\subsection{Cardiomyopathies Associated with Anemia}

Cardiomyopathies associated with anemia are common in Sahel and sub-Saharan Africa. They occur primarily in young women and young children. The anemia is usually severe (30 - $50 \mathrm{~g} / \mathrm{L}$ hemoglobin) and due to a variety of causes: visceral or puerperal hemorrhage, parasitic disease (e.g., malaria, ankylostomiasis), iron deficiency, hemoglobinopathy, or tropical splenomegaly with hypersplenism. The clinical presentation is that of heart failure with increased cardiac output. Left ventricular contractile function often shows only a moderate decrease on echocardiography. The clinical course is most often favorable when the anemia is corrected.

The genetic forms of sickle-cell anemia SS occur in most African countries where their prevalence can reach, in some countries, more than $20 \%$ of the population. Note that sickling and vascular obstruction, an expected complication of sickle-cell anemia, have no effect on myocardial function or coronary circulation [15]. Myocardial perfusion abnormalities were revealed by thallium-201 scintigraphy [16], but echocardiographic studies on sickle-cell anemia patients showed ventricular dilatation with preserved global and segmental contractility [17]. In many patients, a thickening of the ventricular walls and diastolic dysfunction correlated with the time since the onset of disease $[17,18]$. Neither hydroxyurea presumptive antirelapse therapy nor correcting the anemia has any effect on echocardiographic parameters. This parietal hypertrophy could explain the myocardial perfusion abnormalities observed in the scintigraphy studies.

\subsection{Nutritional Cardiomyopathies}

Nutritional cardiomyopathies occur in Southeast Asia in populations on rice-based diets, displaced populations, and prisoners. Beriberi heart disease is the best example [19]. It is associated with thiamine (vitamin B1) deficiency. It is a disease of poverty sometimes aggravated by chronic alcoholism [20]. The vitamin deficiency leads first to a systemic vasodilation, which causes increased cardiac output. As in the case of anemia-associated cardiomyopathies, a substantial increase in cardiac output is associated with hyperkinetic ventricular walls findings by echocardiography. In the most severe forms, the contractile function is drastically impaired and patients go into shock with oligo-anuric renal failure, mimicking Shoshin beriberi as reported by Japanese authors. Thiamine deficiency is frequently accompanied by neuronlogical damage such as peripheral polyneuropathy or Gayet-Wernicke encephalopathy. The diagnosis is often 
based on the context and clinical presentation. The treatment is based on parenteral thiamine supplementation (500 mg/day) followed by oral administration as soon as hemodynamic stability is restored. Without this treatment, standard heart failure therapy is ineffective and the mortality rate high.

\subsection{Parasitic Myocardial Damage}

\subsubsection{Helminthiasis}

Tapeworm larvae (of Taenia solium or Taenia multiceps), as well as eggs of Bilharzia, lung fluke (Paragonimus ringeri) or intestinal fluke (Heterophies heterophies) can be found in the myocardium. Such findings are generally fortuitous and no causal link between their presence and the occurrence of cardiac complications has been shown. The mechanism of heart damage during helminthiasis involves the destructive role of eosinophil granule constituents. This is the case for some endemic tropical helminthiases (schistosomiasis, filariasis) thought to be associated with EMF in west and central African countries, and for other helminthiases (trichinosis, toxocariasis) that cause severe eosinophilia and rare cases of acute myocarditis.

During such a cardiopathy, activated eosinophils mediate cytotoxicity because of the basic proteins contained in their cytoplasmic granules (cationic protein and Major Basic Protein). These proteins, produced in substantial quantity, have a toxic effect on the parasites but also on the tissues. The central nervous system and the heart are the main organs affected [21,22]. Cationic protein has been shown to have a procoagulant activity. The eosinophils, whose production and life span are regulated by cytokines (IL-5, IL-3, GM-CSF), produce interleukin-5, interferon-alpha and TGF-alpha, which stimulate fibroblasts and mediate collagen deposition. The identification, in cardiac biopsies, of degranulated eosinophils, Major Basic Protein, and cationic protein lends support to polynuclear eosinophil cytotoxicity playing a role in the pathogenesis of heart damage [23].

\subsubsection{Protozooses}

\section{1) Malaria}

Impaired cardiac output, increased circulating concentration of cardiac proteins (NT-pro BNP, troponin-T, myoglobin and creatine kinase muscle-brain fraction) were reported in severe falciparum malaria $[24,25]$. These findings have not yet been linked to cardiac dysfunction and they need further investigation. However, more serious complications such as pulmonary edema can occur during cerebral malaria. This pulmonary edema does not have a cardiac origin (pulmonary capillary pressure remains normal). It is caused by an acute respiratory distress syndrome or secondary to fluid overload induced by hypertonic perfusion which should be avoided during treatment of severe falciparum malaria [26].

\section{2) Trypanosomiasis}

The clinical presentation of human American trypanosomiasis or Chagas disease has two variants. The first is early-stage acute myocarditis, often latent, at the time of initial infection. The second is late-stage chronic cardiomyopathy, which is the reason why Chagas disease is such a serious cardiomyopathy. Manifestations include conduction abnormalities, non-specific repolarization changes, ventricular arrhythmia that can be revealed by sudden death, and alterations in global and regional left ventricular contractility. Half or more of the symptomatic patients have a left ventricular apical aneurysm and other segmental contractile abnormalities similar to those seen in coronary heart disease [27]. Ventricular dysfunction and apical aneurysm explain the high prevalence of cardioembolic stroke in Chagas disease in endemic countries [28,29].

The parasite can be detected in cardiac myocytes but the low parasite load suggests that it cannot be the direct cause of the disease: there are several arguments in favor of myocarditis associated with conflicting immunological reactions involving parasite compounds and heart proteins. The blood of patients contains antibodies to myosin, a key protein in cardiac cell contractility. These antibodies react not only with myosin, but also with Trypanosoma chagasi protein $\mathrm{B} 13$. In the myocardium, the identification of lymphocyte infiltrates lends support to the hypothesis that an auto-immune reaction could be involved. Structural similarity has been observed between the cardiac receptors that stimulate the cardiac rhythm in response to adrenalin and protein PO from parasite ribosomes. These arguments are still not sufficient to assert that Chagas disease cardiomyopathy is a parasiteinduced auto-immune disease. A particular difficulty is determining whether the detected antibodies are the cause or the consequence of heart damage.

The heart damage of human African trypanosomeasis has been known since the first clinical descriptions of Le Dantec, but data remain fragmented. The mechanism of heart damage probably has an auto-immune origin like in Chagas disease. Heart damage is most often diagnosed upon a first, severe manifestation that can be fatal (acute pulmonary edema, syncope, cardiac arrest). In the absence of complications, there is a dearth of clinical signs: muffled heart sounds and paradoxical tachycardia in late-stage patients who present with bradycardia due to autonomic nervous system damage. Cardiac enlargement has been described in one third of cases. Electrocardiographic abnormalities, observed in half the patients at all stages of the disease, are low voltage, conduction abnormalities (atrioventricular block and branch block), repolarization abnormalities (ST-T abnormalities, T- 
wave flattening), and prolonged QT interval induced by trypanocide treatment. Conduction abnormalities fluctuate over time and, in some cases, regress with corticosteroid treatment [31,32]. The link between Trypanosoma brucei gambiense infection and African primary cardiomyopathies has been discussed because elevated serum levels of anti-trypanosome antibodies have been observed in DCM patients in Cameroon [30].

\subsection{Primary Cardiomyopathies}

\subsubsection{Dilated Cardiomyopathy (DCM)}

Dilated cardiomyopathy (DCM) is observed in 20\% to $50 \%$ of patients hospitalized for heart failure [31-33]. It affects a majority of men between the ages of 30 and 40 . The diagnosis of primary DCM is a challenge in the absence of coronary angiography or endomyocardial biopsy. These invasive tests are seldom available in developing countries, therefore DCM is often diagnosed after excluding other known causes and external risk factors, and based on compatible history, clinical and paraclinical evidence. In addition to infections at the origin of inflammatory processes (e.g., coxsackievirus, HIV), multiple and sometimes intertwined mechanisms can be involved, such as auto-immune disease, iron overload, alcoholism, malnutrition, and selenium deficiency [20,32, 34]. Alcohol probably plays a substantial role, given the considerable increase in beer consumption in intertropical Africa in the past three decades. Thus, alcohol might be the cause of nearly $45 \%$ of DCM cases in Nigeria. This scourge is all the more ominous because it is associated in many cases with other addictive behaviors (e.g., cocaine, khat) known for their cardiac toxicity. In rural areas, substantial moonshine consumption is responsible for hemosiderosis in South African Bantu populations. Familial DCM, genetically determined by autosomal dominant or recessive genes, or associated with the $\mathrm{X}$ chromosome, accounts for $30 \%$ of cases. Idiopathic DCM forms have been linked to HLA, DR-1, and DRw10 antigens in South African patients [35].

\subsubsection{Hypertrophic Cardiomyopathy (HCM)}

Hypertrophic cardiomyopathy (HCM) has long been considered rare in sub-Saharan Africa. This myth was put to rest when echocardiography became available in most African countries [36]. HCM is as common as anywhere else, with asymmetric septal, apical, or concentric forms, obstructive or not. It is genetically transmitted with the same autosomal dominant pattern as elsewhere and identical mutations of the genes that code for the sarcomere proteins (e.g., beta-MHC, A797T) [37]. Prevalence is not well known in the absence of large epidemiological studies and in medical practice in the tropics it is not easy to differentiate primary from secondary cardiomyopathies (e.g., arterial hypertension, renal insufficiency, amyloidosis).

\subsubsection{Arrhythmogenic Right Ventricular Dysplasia} (ARVD)

Arrhythmogenic right ventricular dysplasia (ARVD) was first reported in Africa in 2000, 40 years after the first case report [38]. It is characterized by the progressive fibro-fatty replacement of the right ventricle myocardial cells. Apoptosis induces ventricular arrhythmias that cause sudden death. End-stage progression is toward right ventricular systolic dysfunction with refractory heart failure. Late-stage, left ventricular involvement can occur. A familial origin can be established in nearly half the cases. Inheritance is autosomal dominant with variable penetrance. The only large study conducted in South Africa by Watkins et al. on 50 patients failed to show any features specific to the study population. The clinical profile and progression are similar to elsewhere with identical mutations of the plakophilin-2 gene [39].

\subsubsection{Left Ventricular Noncompaction (LVNC)}

Left ventricular noncompaction (LVNC), a recently recognized entity, has been reported in African patients. It arises from a lack of ventricular myocardium compaction during embryo development. It results in the persistence of trabeculations and intertrabecular recesses, which contribute to left ventricular dilatation and hypokinesis [40].

\subsubsection{Takotsubo Syndrome}

Takotsubo syndrome was originally described as a stressinduced dysfunction of the left ventricular apex. The pathogenesis is likely to be catecholamine mediated myocyte damage and microvascular dysfunction; however, other alternative theories have been suggested including oxidative stress, transient coronary obstruction, and estrogen deficiency. The clinical presentation is similar to that of an acute myocardial infarction with chest pains and broad T-wave inversions sometimes preceded by ST-segment elevation leading to an initial diagnosis of acute myocardial infarction. So far it has not been described in the tropics, but cases have been reported in the United States among African-Americans $[41,42]$.

\section{Nosology of Tropical Cardiomyopathies}

With the diagnostic tools now available in most tropical countries, as well as genomics and molecular biology advances, it is clear that tropical cardiomyopathies don't differ much from those seen elsewhere. This is true for primary cardiomyopathies (e.g., DCM, HCM, ARVC/D) and the majority of secondary cardiomyopathies. Nutritional cardiomyopathies present no specific clinical features except for environmental factors which explain the 
higher prevalence in malnourished populations. PPCM differs from what is observed elsewhere by a high prevalence and a high mortality rate. Parasite-associated cardiomyopathies apparently boil down to myocardial injury from trypanosomiasis and the deleterious effects of parasite-associated hypereosinophilia. EMF, which is not very different from Löeffler's endocarditis, would simply be the end stage of prolonged hypereosinophilia induced by helminth infection in predisposed patients.

In this context, the concept of tropical cardiomyopathy introduced by Shaper in 1967 is not really relevant. Most of cardiomyopathies seen in tropical countries fit in the classification proposed in 2008 by the European Society of Cardiology, which reflects a simpler approach [43]. The new definition for cardiomyopathy is as follows: " $A$ myocardial disorder in which the heart muscle is structurally and functionally abnormal, in the absence of coronary artery disease, hypertension, valvular disease, and congenital heart disease." This definition excludes secondary cardiomyopathies of known etiology and channelopathies. It retains only five cardiomyopathy types identified according to their morphological phenotype (HCM, DCM, ARVC/D, RCM, and unclassified cardiomyopathy), whether or not they are familial and whether or not the heart is the sole organ affected by the disease.

\section{Clinical Implications}

Given that most of the cardiomyopathies seen in tropical countries, mainly in Africa, have no specificities except for a few of them (Chagas disease, PPCM, EMF, nutritional cardiomyopathies), the clinical approach merely needs to be adapted to the local conditions. Wherever diagnostic tests are limited, echocardiography should be the first diagnostic step. It yields morphological data that allows distinction between DCM, HCM, and RCM. Next, the prevalent or potentially curable tropical cardiomyopathy causes must be excluded. These include anemia, nutritional deficiencies, or HIV infection for DCM; hypertension, renal insufficiency for HCM; and EMF for RCM. A primary cardiomyopathy diagnosis may then be considered and may justify, depending on available means, a more thorough etiological and pathological investigation. The treatment follows the same rules as elsewhere, although in most tropical countries it is not possible for patients to consider an implantable defibrillator, let alone a transplantation [44].

\section{Acknowledgments}

Mrs Khatton.C for her assistance in the translation.

\section{REFERENCES}

[1] J. E. Touze, J. P. Bounhoure, L. Fourcade, C. Jaffiol and
D. Thomas, "Les Facteurs de Risque Cardiovasculaire dans les Pays en Développement: Evolution et Enjeux," Bulletin de L'Académie Nationale de Médecine, Vol. 195, 2011, pp. 1285-1288.

[2] E. Bertrand, "Les Cardiomyopathies en Zone Tropicale,” Annales de Cardiologie et d Angéiologie, Vol. 35, 1986, pp. 305-310.

[3] A. G. Shaper, "On the Nature of Some Tropical Cardiomyopathies," Transactions of the Royal Society of Tropical Medicine and Hygiene, Vol. 61, No. 4, 1967, pp. 458481. http://dx.doi.org/10.1016/0035-9203(67)90097-1

[4] WHO, "Report of the 1995 World Health Organization/International Society and Federation of Cardiology Task force on the Definition and Classification of Cardiomyopathies," Circulation. Vol. 93, 1996, pp. 841-842. http://dx.doi.org/10.1161/01.CIR.93.5.841

[5] B. J. Maron, J. A. Towbin, G. Thiene, C. Antzelevitch, D. Corrado, D. Arnett, A. J. Moss, C. E. Seidman and J. B. Young, "Contemporary Definitions and Classification of the Cardiomyopathies: An American Heart Association Scientific Statement From the Council on Clinical Cardiology, Heart Failure and Transplantation Committee; Quality of Care and Outcomes Research and Functional Genomics and Translational Biology Interdisciplinary Working Groups; and Council on Epidemiology and Prevention," Circulation. Vol. 113, 2006, pp. 1807-1816. http://dx.doi.org/10.1161/CIRCULATIONAHA.106.1742 $\underline{87}$

[6] A. O. Falase, "Endomyocardial Fibrosis in Africa," Postgraduate Medical Journal, Vol. 59, No. 689, 1983, pp. 170-177. http://dx.doi.org/10.1136/pgmj.59.689.170

[7] J. Freers, J. Amandua, R. Mugerwa and C. Sezi, "Endomyocardial Fibrosis and Eosinophilia,” Lancet, Vol. 342, No. 8881, 1993, pp. 1233-1234. http://dx.doi.org/10.1016/0140-6736(93)92211-B

[8] J. J. Andy, P. O. Ogunowo, N. A. Akpan, C. O. Odigwe, I. A. Ekanem and R. A. Esin, "Helminth Associated Hypereosinophilia and Tropical Endomyocardial Fibrosis (EMF) in Nigeria,” Acta Tropica, Vol. 69, No. 2, 1998, pp. 127-140.

http://dx.doi.org/10.1016/S0001-706X(97)00125-3

[9] C. L. Sezi, "Effect of Protein Deficient Cassava Diet on Cercopithecus Aethiops Hearts and Its Possible Role in the Aetiology and Pathogenesis of Endomyocardial Fibrosis in Man," East African Medical Journal, Vol. 73, Suppl. 5, 1996, pp. S11-16.

[10] D. Metras, A. O. Coulibaly and K. Ouattara, "The Surgical Treatment of Endomyocardial Fibrosis: Results in 55 Patients,” Circulation, Vol. 72, 1985, pp. 274-279.

[11] D. Metras, A. Q. Coulibaly and K. Ouattara, "Recent Trends in the Surgical Treatment of Endomyocardial Fibrosis," The Journal of Cardiovascular Surgery, Vol. 28, 1987, pp. 607-613.

[12] K. Tibazarwa and K. Sliwa, "Peripartum Cardiomyopathy in Africa: Challenges in Diagnosis, Prognosis, and Therapy,” Progress in Cardiovascular Diseases, Vol. 52, No. 4, 2010, pp. 317-325.

http://dx.doi.org/10.1016/j.pcad.2009.11.003

[13] H. Yamac, I. Bultmann, K. Sliwa and D. Hilfiker-Kleiner 
"Prolactin: A New Therapeutic Target in Peripartum Cardiomyopathy," Heart, Vol. 96, No. 17, 2010, pp. 13521357. http://dx.doi.org/10.1136/hrt.2009.179218

[14] K. Sliwa, D. Hilfiker-Kleiner, M. C. Petrie, A. Mebazaa, B. Pieske, E. Buchmann, V. Regitz-Zagrosek, M. Schaufelberger, L. Tavazzi, D. J. van Veldhuisen, H. Watkins, A. J. Shah, P. M. Seferovic, U. Elkayam, S. Pankuweit, Z. Papp, F. Mouquet and J. J. McMurray, "Current State of Knowledge on Aetiology, Diagnosis, Management, and Therapy of Peripartum Cardiomyopathy: A Position Statement from the Heart Failure Association of the European Society of Cardiology Working Group on peripartum cardiomyopathy,” European Journal of Heart Failure, Vol. 12, No. 8, 2010, pp. 767-778. http://dx.doi.org/10.1093/eurjhf/hfq120

[15] W. Covitz, M. Espeland, D. Gallagher, W. Hellenbrand, S. Leff and N. Talner, "The Heart in Sickle Cell Anemia: The Cooperative Study of Sickle Cell Disease (CSSCD)," Chest, Vol. 108, No. 5, 1995, pp. 1214-1219. http://dx.doi.org/10.1378/chest.108.5.1214

[16] P. Acar, C. Maunoury, M. de Montalembert and Y. Dulac, "Anomalies de la Perfusion Myocardique dans la Drépanocytose de L'enfant: Étude par la Tomoscintigraphie Myocardique," Archives des Maladies du Coeur et des Vaisseaux, Vol. 96, 2003, pp. 507-510.

[17] T. Mardelle, A. Ekra and E. Bertrand, "LV Function in Sickle Cell Anemia,” American Heart Journal, Vol. 12, No. 6, 1986, pp. 1356-1357. http://dx.doi.org/10.1016/0002-8703(86)90403-5

[18] A. C. Eddine, O. Alvarez, S. E. Lipshultz, R. Kardon, K. Arheart and S. Swaminathan, "Ventricular Structure and Function in Children with Sickle Cell Disease Using Conventional and Tissue Doppler Echocardiography," American Journal of Cardiology, Vol. 109, No. 9, 2012, pp. 1358-1364. http://dx.doi.org/10.1016/j.amjcard.2012.01.001

[19] T. L. Miller, D. Neri, J. Extein, G. Somarriba and N. Strickman-Stein, "Nutrition in Pediatric Cardiomyopasthy,” Progress in Pediatric Cardiology, Vol. 24, No. 1, 2007, pp. 59-71.

http://dx.doi.org/10.1016/j.ppedcard.2007.08.007

[20] S. L. Tobias, J. van der Westhuyzen, R. E. Davis, G. C. Icke and P. M. Atkinson, "Alcohol Intakes and Deficiencies in Thiamine and Vitamin B6 in Black Patients with Cardiac Failure,” South African Medical Journal, Vol. 76, 1989, pp. 299-302.

[21] M. H. Roehrl, M. P. Alexander, S. B. Hammond, M. Ruzinova, J. Y. Wang and C. J. O’Hara, "Eosinophilic Myocarditis in Hypereosinophilic Syndrome. American Journal of Hematology, Vol. 86, No. 7, 2011, pp. 607608. http://dx.doi.org/10.1002/ajh.21943

[22] P. U. Ogbogu, D. R. Rosing and M. K. Horne, "Cardiovascular Manifestations of Hypereosinophilic Syndromes," Immunology And Allergy Clinics of North America, Vol. 27, No. 3, 2007, pp. 457-475. http://dx.doi.org/10.1016/j.iac.2007.07.001

[23] B. L. Wright, K. M. Leiferman and G. J. Gleich, "Eosinophil Granule Protein Localization in Eosinophilic Endomyocardial Disease," The New England Journal of
Medicine, Vol. 365, 2011, pp. 187-188. http://dx.doi.org/10.1056/NEJMc1103005

[24] S. Yacoub, H. J. Lang, M. Shebbe, et al., "Cardiac Function and Hemodynamics in Kenyan Children with Severe Malaria,” Critical Care Medicine, Vol. 38, No. 3, 2010, pp. 940-945.

http://dx.doi.org/10.1097/CCM.0b013e3181cd114a

[25] WHO, "Severe and Complicated Falciparum Malaria," Transactions of the Royal Society of Tropical Medicine and Hygiene, Vol. 94, Suppl. 1, 2000, pp. 1-90.

[26] H. Acquatella, "Echocardiography in Chagas Disease," Circulation, Vol. 115, 2007, pp. 1124-1131. http://dx.doi.org/10.1161/CIRCULATIONAHA.106.6273 $\underline{23}$

[27] S. Yacoub, A. O. Mocumbi and M. H. Yacoub, "Neglected Tropical Cardiomyopathies (I): Chagas Disease," Heart, Vol. 94, No. 2, 2008, pp. 244-248. http://dx.doi.org/10.1136/hrt.2007.132316

[28] F. J. Carod-Artal, “'Trypanosomiasis, Cardiomyopathy and the Risk of Ischemic Stroke," Expert Review of Cardiovascular Therapy, Vol. 8, No. 5, 2010, pp. 717-728. http://dx.doi.org/10.1586/erc.10.33

[29] J. A. Blum, M. J. Zellweger, C. Burri and C. Hatz, "Cardiac Involvement in African and American Trypanosomiasis,” The Lancet Infectious Diseases, Vol. 8, No. 10, 2008, pp. 631-641. http://dx.doi.org/10.1016/S1473-3099(08)70230-5

[30] E. Bertrand, "L'atteinte Cardiaque dans la Trypanosomiase Africaine,” Médecine Tropicale, Vol. 47, 1987, pp. 91-93.

[31] K. Blackett and J. L. Ngu, "Immunological Studies in Congestive Cardiomyopathy in Cameroon,” British Heart Journal, Vol. 38, 1976, pp. 605-611. http://dx.doi.org/10.1136/hrt.38.6.605

[32] K. Sliwa, A. Damasceno and B. M. Mayosi, "Epidemiology and Etiology of Cardiomyopathy in Africa,” Circulation, Vol. 112, 2005, pp. 3577-3583. http://dx.doi.org/10.1161/CIRCULATIONAHA.105.5428 $\underline{94}$

[33] B. Maharaj, "Causes of Congestive Heart Failure in Black Patients at King Edward VIII Hospital, Durban,” Cardiovascular Journal of South Africa, Vol. 2, 1991, pp. 3132.

[34] A. G. Amoah, C. Kallen, "Aetiology of Heart Failure as Seen from a National Cardiac Referral Centre in Africa," Cardiology, Vol. 93, 2000, pp. 11-18. http://dx.doi.org/10.1159/000006996

[35] B. Maharaj, M. G. Hammond, "HLA-A, B, DR, and DQ Antigens in Black Patients with Idiopathic Dilated Cardiomyopathy," American Journal of Cardiology, Vol. 65, No. 20, 1990, pp. 1402-1403.

http://dx.doi.org/10.1016/0002-9149(90)91337-6

[36] B. Abegaz, "The Impact of Echocardiography in the Diagnosis of Hypertrophic Cardiomyopathy,” East African Medical Journal, Vol. 67, 1990, pp. 556-567.

[37] J. C. Moolman-Smook, B. M. Mayosi, P. A. Brink and V. A. Corfield, "Molecular Genetics of Cardiomyopathy: Changing Times, Shifting Paradigms," Cardiovascular 
Journal of South Africa, Vol. 14, 2003, pp. 145-155.

[38] M. J. Munclinger, J. J. Patel and A. S. Mitha, "Follow-Up of Patients with Arrhythmogenic Right Ventricular Cardiomyopathy Displasia,” South African Medical Journal, Vol. 90, 2000, pp. 61-68.

[39] D. A. Watkins, N. Hendricks, G. Shaboodien, M. Mbele, M. Parker, B. Z. Vezi, A. Latib, A. Chin, F. Little, M. Badri, J. C. Moolman-Smook, A. Okreglicki and B. M. Mayosi, "Clinical Features, Survival Experience, and Profile of Plakophylin-2 Gene Mutations in Participants of the Arrhythmogenic Right Ventricular Cardiomyopathy Registry of South Africa," Heart Rhythm, Vol. 6, 2009, pp. S10-17.

http://dx.doi.org/10.1016/j.hrthm.2009.08.018

[40] P. Paule, L. Braem, D. Mioulet, B. Jop, A. Théron, J. M. Gil, P. Héno and L. Fourcade, "La non Compaction du Ventricule Gauche, une Cardiomyopathie du Sujet Jeune: Premières Observations Africaines," Médecine Tropicale, Vol. 67, 2007, pp. 587-593.

[41] A. Qaqa, J. Daoko, N. Jallad, O. Aburomeh, I. Goldfarb and F. Shamoon, "Takotsubo Syndrome in African
American vs. Non-African American Women,” Western Journal of Emergency Medicine, Vol. 12, 2011, pp. 218223.

[42] H. M. Patel, B. K. Kantharia, D. L. Morris and S. Yaz-danfar, "Takotsubo Syndrome in African-American Women with Atypical Presentations: A Single Center Experience,” Clinical Cardiology, Vol. 30, No. 1, 2007, pp. 14-18. http://dx.doi.org/10.1002/clc.21

[43] P. Elliott, B. Andersson, E. Arbustini, Z. Bilinska, F. Cecchi, P. Charron, O. Dubourg, U. Kühl, B. Maisch, W. J. McKenna, L. Monserrat, S. Pankuweit, C. Rapezzi, P. Seferovic, L. Tavazzi and A. Keren, "Classification of the Cardiomyopathies: A Position Statement from the European Society of Cardiology Working Group on Myocardial and Pericardial Diseases,” European Heart Journal, Vol. 29, No. 2, 2008, pp. 270-276. http://dx.doi.org/10.1093/eurheartj/ehm342

[44] R. N. Millar and B. M. Mayosi, "Utilization of Implantable Defibrillators in Africa,” Cardiac Electrophysiology Review, Vol. 7, No. 1, 2003, pp. 14-16. http://dx.doi.org/10.1023/A:1023622502931 